\title{
Castleman Disease in Children from Histopathology to Therapy
}

\author{
Asmaa Hamoda ${ }^{1,2}$, Hanaa Rashad ${ }^{1}$, Ola Ahmad ${ }^{3}$, Hala Reda ${ }^{4,5}$, Iman Zaki ${ }^{6,7}$, Naglaa Elkinaai ${ }^{8,9}$, \\ Mohamed Sedki ${ }^{1,10}$, Alaa El Hadad ${ }^{1,2}$, Samah Semary ${ }^{1,11, *}$ \\ ${ }^{1}$ Department of Pediatric Oncology, Children Cancer Hospital Egypt, Cairo, Egypt \\ ${ }^{2}$ Department of Pediatric Oncology, National Cancer Institute, Cairo University, Cairo, Egypt \\ ${ }^{3}$ Department of Clinical Research, Children Cancer Hospital Egypt, Cairo, Egypt \\ ${ }^{4}$ Department of Clinical Pathology, Children Cancer Hospital Egypt, Cairo, Egypt \\ ${ }^{5}$ Department of Clinical Pathology, National Cancer Institute, Cairo University, Cairo, Egypt \\ ${ }^{6}$ Department of Radiodiagnosis, National Cancer Institute, Cairo University, Cairo, Egypt \\ ${ }^{7}$ Department of Radiodiagnosis, Children Cancer Hospital Egypt, Cairo, Egypt \\ ${ }^{8}$ Department of Pathology, National Cancer Institute, Cairo University, Cairo, Egypt \\ ${ }^{9}$ Department of Pathology, Children Cancer Hospital Egypt, Cairo, Egypt \\ ${ }^{10}$ Department of Pediatrics, National Research Center, Cairo, Egypt \\ ${ }^{11}$ Department of Clinical Oncology, Beni-suef University, Beni-suef, Egypt
}

\section{Email address:}

samahsemary@yahoo.com (S. Semary),samah.semary@57357.org (S. Semary)

${ }^{*}$ Corresponding author

\section{To cite this article:}

Asmaa Hamoda, Hanaa Rashad, Ola Ahmad, Hala Reda, Iman Zaki, Naglaa Elkinaai, Mohamed Sedki, Alaa El Hadad, Samah Semary. Castleman Disease in Children from Histopathology to Therapy. Cancer Research Journal. Vol. 8, No. 4, 2020, pp. $100-103$. doi: $10.11648 /$ j.crj.20200804.16

Received: April 11, 2020; Accepted: December 15, 2020; Published: December 22, 2020

\begin{abstract}
Background: Castleman disease (CD) describes a group of rare lymphoproliferative disorder with characteristic histopathology. It presents with heterogeneous clinical features whether unicentric (UC) or multicentric disease (MCD). The aim of the work was to describe clinic-pathological characteristics, management and outcome of different types of castleman disease in CCHE. It is a retrospective study, all children with (CD) treated from July 2007 till end of 2017 were analyzed as regard diagnosis, management and outcome. The results showed that, twelve patients with a median age of 11.5 years (ranging from 4 - to 17 years) were enrolled. Eight of them $(66.6 \%)$ were males and $4(33.3 \%)$ were females $(33.3 \%)$. Histopathology was either hyaline vascular in 8 patients $(66.6 \%)$ or plasma cell variant in 4 patients $(33.3 \%)$. Nine patients $(75 \%)$ had unicentric disease (UC) with lymphadenopathy. Three patients (25\%) were multi-centric disease (MCD); out of them one patient had immune bi-cytopenia, small intestinal thickening and splenomegaly and another one had extensive pulmonary involvement and respiratory distress, while the third patient had pleural effusion and ascites. HIV antibody was negative for all patients. Regarding the three MCD patients, IL-6 was normal in one patient, and extremely high in second one, while it hasn't been done for the third patient. All UC patients underwent surgical excision and are alive with no active disease till now. All MCD patients received systemic steroids as initial therapy, followed by R- CHOP (Rituximab-cyclophosphamide-doxorubicinvincristine-prednisone) as salvage therapy, except for one patient with pulmonary involvement who didn't achieved clinical response and received single agent Rituximab with Anti IL-6. Unfortunately this patient is still having uncontrolled systemic manifestation, while the other two patients showed partial response. In conclusion, Uni-centric castleman disease is a localized surgically cured disease. MCD treatment remains challenging, and the outcome is controversial, so Uniform treatment guidelines are mandatory.
\end{abstract}

Keywords: Castleman Disease, Unicentric Disease, Multicentric Disease, Histopathology, Ritoxomab, Anti-IL6 


\section{Introduction}

Castleman disease (CD) is described as a group of heterogeneous hematologic disorders which involve a spectrum of lymph node histopathology consisting of atrophied germinal centers with hypervascularization (hyaline vascular/hypervascular histopathological subtype) on the other hand the histopathology may consisting of hyperplastic germinal centers concomitant with polytypic plasmacytosis (plasmacytic histopathological subtype) \& mixed type [1]. Castleman disease is classified to two groups according to the affected region, Unicentric CD (UCD) affecting a single region of enlarged lymph nodes with diagnostic histopathology and causing mild symptoms, which can be treated with excision of the enlarged lymph node. On the other hand, the multicentric CD (MCD) affecting more than one lymph node group, causing systemic inflammation, multicentric lymphadenopathy with characteristic histopathology, affecting the bone marrow causing cytopenias, and may progressed to be fatal multiple organ dysfunction by the effect of cytokine storm often including interleukin 6 (IL-6) [2]. Histological Variants include, Hyaline vascular variant, Plasma Cell variants of $\mathrm{CD}$, and Mixed type [3].

\section{Aim of the Work}

Was to describe the series of patients with castleman diseases presented to the hospital from July 2007 till end of 2017, look into different treatment modality for MCD patients, trying to implement the guidelines proposed management of MCD according to the Castleman Disease Collaborative Network (CDCN).

\section{Patients and Methods}

A retrospective study included all patients diagnosed as Castleman disease either Unicentric or multicentric From July 2007 till end of 2017 at Children Cancer Hospital Egypt. Patients were included if they were less than 18 years, no previous chemotherapy,

Pathology confirmed Castleman disease, CBC, Kidney \& liver functions, CRP, IL6, HIV PCR, and HHV8 if applicable were done for them. CT Whole body, Echo was done initially for all the study group patients to classify the disease as unicentric or multicentric, finally line of treatment received was mentioned.

Histopathology:

Ten out of twelve cases show hyaline vascular castleman disease.

Hyaline vascular Castleman disease showed prominent hyalinized walls proliferating vessels. Oninon skin pattern with thickened mantle zones and ring like lymphocyte arrangement are frequently noted. The germinal centres showed frequent atresia and were often penetrated by hyalinized vessels featuring lollipop like appearance. The interfollicular areas, often showed extensive vascular proliferation with perivascular hyalinization.

Multicentric Castleman disease featured mixture of hyaline vascular and plasma cell pattern, the latter showed sheets of mature plasma cells in the interfollicular areas. The follicles showed hyperplastic germinal centers.

H\& E stained sections showing attretic follicles with thick hyalinized vessels inside and outside the follicles

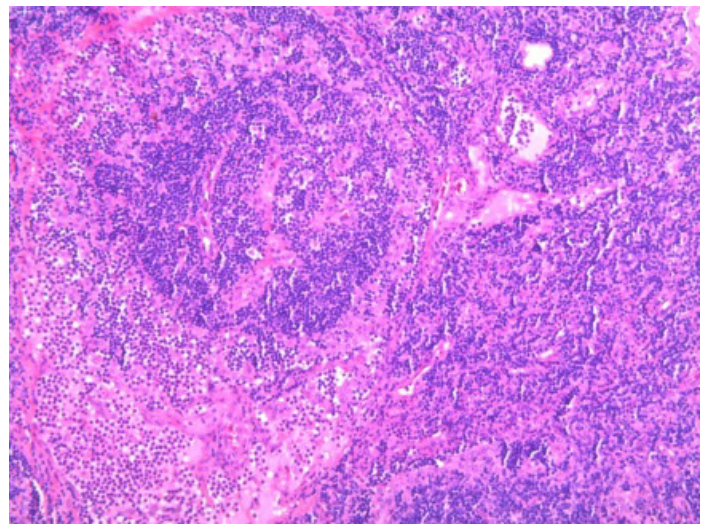

Figure 1. H\& E stained sections showing attretic follicles with thick hyalinized vessels inside and outside the follicles.

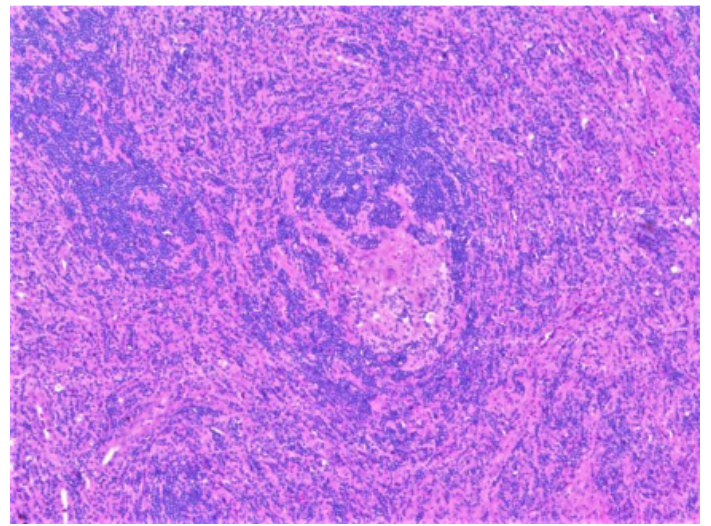

Figure 2. H\& E stained sections showing attretic follicles with thick hyalinized vessels inside and outside the follicles.

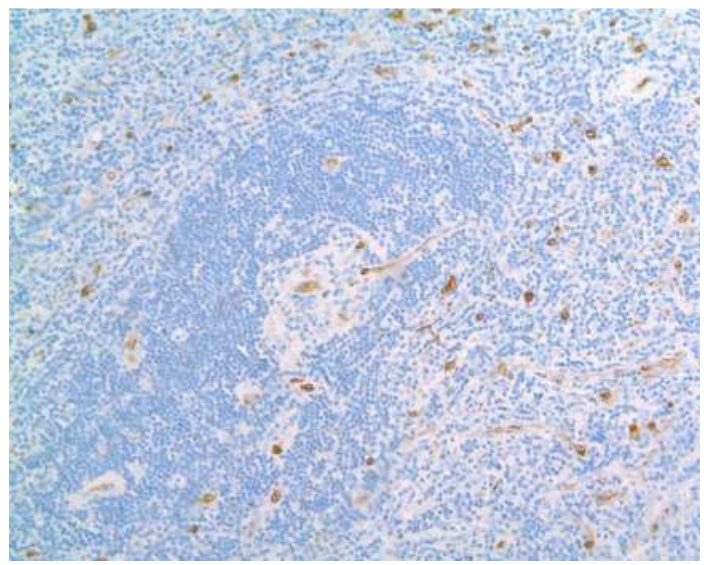

Figure 3. CD34 immunostain showing endothelial cells piercing into the follicle (lollipop sign). 


\section{Results}

A total of 12 patients were enrolled in the current study. The median age was 11.5 years (ranging from 4 - to 17 years). Eight of them $(66.6 \%)$ were males and $4(33.3 \%)$ were females $(33.3 \%)$. Nine of them (75\%) had unicentric disease and the remaining, 3 patients $(25 \%)$ had multicentric disease. Unicentric patients were presented with Lymphadenopathy either Cervical, supraclavicular, Axillary LN, 2 of them were having retroperitoneal and paravertebral soft tissue lesion which was excised completely, the 3 patients who had mulicentric disease were presented with immune Cytopenia (anemia and thrombocytopenia ) with small bowel thickening forming mass and splenomegaly in first patient, respiratory distress with extensive pulmonary involvement in the second one, and the last patient presented with pleural effusion and ascites. Whole body CT scan done for all patient, BMA done only for patients with Cytopenia, viral screening was done in the form of HIV
Antibodies for all patients and it was negative, HHV8 was not available so, not performed, IL6 done only for 2 patients out of the 3 patients $(25 \%)$ who had multicentric disease, it was very high in one of them and was normal in the other patient, histopathology for all patient confirming castlaman either hyaline vascular or plasma cell variant, hyaline vascular in 8 patients $(66.6 \%)$ or plasma cell variant in 4 patients $(33.3 \%)$ table 1. Patients with unicentric disease underwent surgical excision, no further treatment was received, and all of them are alive in complete remission. Patients with Multicentric disease received systemic treatment including Steroids initially with no response, then received single agent Rituximab, with no marked improvement in their conditions, trial of R-CHOP (rituximabcyclophosphamide-doxorubicin-vincristine-prednisone) was done, lastly Anti-IL6 was tried. They improved much apart from the patient with pulmonary involvement still with uncontrolled systemic manifestation and respiratory distress.

Table 1. Characteristics of The Studied Population.

\begin{tabular}{lc}
\hline age & median age: 11.5 years \\
\hline sex & $8 / 12(66.6 \%)$ \\
male & $4 / 12(33.3 \%)$ \\
female & $8 / 12(66.6 \%)$ \\
Histo-pathological Types: & $4 / 12(33.3 \%)$ \\
Hyaline vascular & $9 / 12(75 \%)$ \\
Plasma cell variant & $3 / 12(25 \%)$ \\
Presentation: & \\
Localized/ Unicentric disease & \\
Multicentric disease with: & \\
1- immune bicytopenia, small intestinal thickening and splenomegaly & \\
2- extensive pulmonary involvement and respiratory distress & \\
3- pleural effusion \& ascites & \\
\hline
\end{tabular}

\section{Discussion}

Castleman's disease (CD) is a rare disease, it usually occurs in young adults and rarely in childhood, presented with localized or disseminated, lymphoproliferative disorder [4]. In the current study there were only 12 pediatric patients with confirmed pathology as castleman disease between the periods from July 2007 till end of 2017. Age of onset varied from 0 to 16 years (median 8.5 years) [5]. In the current study, the median age was 11.5 years (ranging from 4 - to 17 years). The majority was in males [5], and as in the current study. Unicentric CD (UCD) is most commonly found in the mediastinum [6], that was against the current study finding as unicentric disease had the upper hand of the study cases (75\%) but Unicentric patients were presented with Lymphadenopathy either Cervical, supraclavicular, Axillary LN, 2 of them were having retroperitoneal and paravertebral soft tissue lesion and the rest had mediastinal masses. In unicentric disease Hyaline vascular pathology subtype is more likely than plasma cell variant $[6,7]$, as in the current study as about 8 patients $(66.6 \%)$ had unicentric disease with Hyaline vascular variant by pathology, that give an impetration of the presence of hyaline vascular variant is with localized less aggressive disease [8], on the other hand the presence of plasma cell variant is with disseminated and more aggressive disease [9], in multicentric disease. Multicentric
Castleman disease presents with systemic symptoms in the form of fever, night sweat, and loss of weight, generalized lymphadenopathy, and laboratory markers of inflammation [10], cytopenias, and multiple organ system faliure [11], as in the current study, the 3 patients who had multicentric disease were presented with immune Cytopenia, disseminated disease with intestinal and pulmonary complication, and splenomegaly. These dissemination and aggression of the disease was due to cytokines as interleukin-6 [12]. A subset of multicentric CD is caused by human herpesvirus-8 (HHV-8-associated MCD), whereas HHV-8-negative MCD cases remain idiopathic (iMCD) [11]. In the current study, HIV Antibodies as viral screening was done for all patients and was negative, HHV8 was not available so, not performed then could not assess its impact on the disease, IL6 done only for 2 patients out of the 3 patients $(25 \%)$ who had multicentric disease, it was significantly high in one and was normal in the other patient. The treatment of unicentric disease is by surgical excision, the prognosis is perfect and disease recurrence is rare $[13,14]$, as in the current study as Patients with unicentric disease underwent surgical excision, no further treatment was needed, and all of them are alive in complete remission. The working group recommends siltuximab (11 $\mathrm{mg} / \mathrm{kg}$ every 3 weeks) with or without corticosteroids as firstline therapy for all patients [15]. Patients who respond to siltuximab should taper off corticosteroids and continue receiving siltuximab monotherapy indefinitely. Treatment of 
patients who do not respond to siltuximab should be tailored by disease severity into non-severe or severe organ dysfunction. Patients with non-severe disease who fail to adequately respond to siltuximab after 3 to 4 doses should receive rituximab ( 375 $\mathrm{mg} / \mathrm{m}^{2} \quad 3 \quad 4-8$ weekly doses) + immunomodulatory/immunosuppressive agent, such as cyclosporine, sirolimus, anakinra, thalidomide, or bortezomib [16].

Among responders, patients with a mild pretreatment disease course may be observed off of treatment, whereas patients with a more intense pretreatment disease course should be maintained on an immunomodulatory agent.

Third-line treatment of patients with non-severe disease that fail to respond to rituximab after 4 to 8 weekly doses involves an immunomodulatory/immunosuppressive agent. For patients with severe disease, accelerated weekly dosing of siltuximab with high-dose corticosteroids (eg, methylprednisolone $500 \mathrm{mg}$ daily) is recommended. Any sign of worsening organ function should immediately trigger the initiation of chemotherapy (eg, rituximab-cyclophosphamide doxorubicin-vincristineprednisone, bortezomib-dexamethasone thalidomideadriamycin-cyclophosphamide-etoposide-rituximab,

cyclophosphamide-etoposide-rituximab) [17]. In the current study, Patients with Multicentric disease received systemic treatment initially they received Steroids unforshinitly with no response, then Rituximab was tried, with no dramatic improvement, followed by giving R-CHOP (rituximabcyclophosphamide-doxorubicin-vincristine-prednisone), lastly Anti-IL6 was tried. All of them improved except the patient who had pulmonary involvement is still with uncontrolled systemic manifestation and has respiratory distress, then MCD treatment remains challenging, and the outcome is controversial, so Uniform treatment guidelines are mandatory.

\section{Conclusion}

Castleman's disease (CD) is a rare disease, it usually occurs in young adults and rarely in childhood. Uni-centric Castleman disease is a localized surgically cured disease. Multicentric disease's treatment remains challenging, and the outcome is controversial, so Uniform treatment guidelines are mandatory.

\section{Acknowledgements}

I gratefully acknowledge my colleagues in NHL study team member in Children Cancer Hospital Egypt, and my patients.

\section{References}

[1] Fajgenbaum dc, van rhee f, nabel cs. Hhv-8-negative, idiopathic multicentric castleman disease: novel insights into biology, Pathogenesis, and therapy. Blood. 2014; 123 (19): 2924-2933.

[2] Liu AY, nabel CS, finkelman BS, et al. Idiopathic multicentricCastleman's disease: a systematic literature review. Lancet haematol. 2016; 3 (4): e163-e175.
[3] Munshi N, mehra $M$, van de velde $H$, desai $A$, potluri $R$, vermeulen $\mathrm{J}$. Use of a claims database to characterize and estimate the incidence rateFor castleman disease. Leuk lymphoma. 2015; 56 (5): 1252-1260.

[4] Piero Farruggia, 1 Antonino Trizzino, 1 Nunzia Scibetta, 2 et al, Castleman's disease in childhood: report of three cases and review of the literature, Ital J Pediatr. 2011; 37: 50.

[5] V. I. Burlakov, A. L. Kozlova, A. J. Shcherbina, et al, THU0587 Clinical Characterization of Castleman's Disease in A Group of Pediatric Patients, Poster Presentations 2016

[6] Seth J. Kligerman, Aaron Auerbach, Teri J. Franks, Castleman Disease of the Thorax: Clinical, Radiologic, and Pathologic Correlation: From the Radiologic Pathology Archives, RadioGraphicsVol. 36, No. 5, 2016.

[7] Weisenburger DD, Nathwani BN, Winberg CD, Rappaport H. Multicentric angiofollicular lymph node hyperplasia: a clinicopathologic study of 16 cases. Hum Pathol. 1985; 16: 162-72. doi: 10.1016/S0046-8177(85)80065-4.

[8] Frizzera G, Peterson BA, Bayrd ED, Goldman A. A systemic lymphoproliferative disorder with morphologic features of Castleman's disease: clinical findings and clinicopathologic correlations in 15 patients. J Clin Oncol. 1985; 3 (9): 1202-16.

[9] Kasantikul V, Panyavoravut V, Benjavongkulchai S, Panichabhongse V. Castleman's disease: a clinicopathologic study of 12 cases. J Med Assoc Thai. 1997; 80 (3): 195-201.

[10] Sarra Benmiloud, Sana Chaouki, Samir Atmani, et al, Multicentric Castleman's Disease in a Child Revealed by Chronic Diarrhea, Case Reports in Pediatrics, Volume 2015, Article ID 689206, 4 pages.

[11] David C. Fajgenbaum, Thomas S. Uldrick, Adam Bagg, et al, International, evidence-based consensus diagnostic criteria for HHV-8-negative/idiopathic multicentric Castleman disease, Blood $2017 \quad 129$ : 1646-1657; doi: https://doi.org/10.1182/blood-2016-10-746933.

[12] Brandt SJ, Bodine DM, Dunbar CE, Nienhuis AW. Dysregulated interleukin expression produces a syndrome resembling Castleman's disease in mice. J Clin Invest. 1990; 86 (2): 592-9. doi: 10.1172/JCI114749.

[13] Ye B, Gao SG, Li W et al. A retrospective study of unicentric and multicentric Castleman's disease: a report of 52 patients. Med Oncol 2010; 27 (4): 1171-1178.

[14] Talat N, Belgaumkar AP, Schulte KM. Surgery in Castleman's disease: a systematic review of 404 published cases. Ann Surg 2012; 255 (4): 677-684.

[15] Summerfield GP, Taylor W, Bellingham AJ, Goldsmith HJ. Hyaline-vascular variant of angiofollicular lymph node hyperplasia with systemic manifestations and response to corticosteroids. J Clin Pathol. 1983; 36 (9): 1005-11. doi: 10.1136/jcp.36.9.1005.

[16] Bower M, Veraitch O, Szydlo R, Charles P, Kelleher P, Gazzard B, Nelson M, Stebbing J. Cytokine changes during rituximab therapy in HIV-associated multicentric Castleman disease. Blood. 2009; 113 (19): 4521-4. doi: 10.1182/blood2008-12-197053.

[17] David Green, MD, PhD reviewing Van Rhee $\mathrm{F}$ et al, Treatment Guidelines for Idiopathic Multicentric Castleman Disease, Blood 2018. 